\title{
Search for vacancies in concentrated solid-solution alloys with fcc crystal structure
}

\author{
L. Resch $\odot,{ }^{1,}{ }^{*}$ M. Luckabauer $\odot,{ }^{2}$ N. Helthuis $\odot,{ }^{2}$ N. L. Okamoto $\odot,{ }^{3}$ T. Ichitsubo $\odot,{ }^{3}$ R. Enzinger $\odot,{ }^{1}$ \\ W. Sprengel ${ }^{1}{ }^{1}$ and R. Würschum ${ }^{1}{ }^{1}$ \\ ${ }^{1}$ Institute of Materials Physics, Graz University of Technology, Petersgasse 16, 8010 Graz, Austria \\ ${ }^{2}$ Faculty of Engineering Technology, University of Twente, P.O. Box 217, 7500AE Enschede, The Netherlands \\ ${ }^{3}$ Institute for Materials Research, Tohoku University, Sendai 980-8577, Japan
}

(Received 25 February 2020; accepted 4 May 2020; published 22 June 2020)

\begin{abstract}
Single-phase concentrated solid-solution alloys (CSA), i.e., alloys without a principle alloying element but one randomly populated crystal structure, exhibit attractive material properties such as very high ductility at cryogenic temperatures, a gentle decrease of strength with temperature, or an unexpectedly high resistance against irradiation. For clarification of those observations assessment of atomic transport mechanisms including formation and migration of equilibrium point defects is indispensable. Positron annihilation lifetime spectroscopy measurements are performed to quantify the concentration of quenched-in thermal vacancies in fcc CSAs after quenching from temperatures close to their onset of melting. For various alloy compositions the concentration of quenched-in vacancies decreases with increasing entropy of mixing $\Delta S_{\text {mix }}$. Whereas alloys with three constituents in nonequimolar fractions ( $\mathrm{CrFeNi}$ ) exhibit vacancy concentrations in the $10^{-5}$ range, the studied alloys with four $(\mathrm{CoCrFeNi})$ and five constituents $(\mathrm{CoCrFeMnNi}, \mathrm{AlCoCrFeNi})$ do not show a vacancy-specific positron lifetime. Therefore, the concentration of quenched-in vacancies must be in the range of $10^{-6}$ or less. It can be concluded that there is either only a vanishingly small fraction of vacancies present at temperatures near the onset of melting or the generated vacancies are inherently unstable.
\end{abstract}

DOI: 10.1103/PhysRevMaterials.4.060601

\section{INTRODUCTION}

Over the last decade a new class of multicomponent singlephase alloys, often referred to as high entropy alloys, or concentrated solid-solution alloys, gained increasing attention (see, e.g., Refs. [1-4]). Contrary to conventional alloys, which consist of one base element and minor additions of other elements to achieve desired properties, these concentrated solid-solution alloys consist of multiple principal elements in equimolar fractions. Based on general knowledge on physical metallurgy, multicomponent alloys were assumed to form multiple phases and intermetallic compounds and to experience undesired properties such as brittleness. However, it turned out that a high number of different multicomponent alloys can be designed which form random solid solutions with mostly face-centered-cubic or body-centered-cubic structures. Concentrated solid-solution alloys have been reported to possess various desirable material properties such as high strength in combination with good ductility even at high temperatures [5,6], excellent wear and corrosion resistance [7,8], as well as superior radiation tolerance $[9,10]$. These properties make them interesting candidates for components used in extreme environments such as, for example, nuclear reactors. The enhanced radiation tolerance of concentrated solid-

\footnotetext{
*Corresponding author: 1.resch@tugraz.at
}

Published by the American Physical Society under the terms of the Creative Commons Attribution 4.0 International license. Further distribution of this work must maintain attribution to the author(s) and the published article's title, journal citation, and DOI. solution alloys has been the subject of several studies which concluded that the most probable cause is the distortion of the crystal lattice which slows down dislocation movement [10]. Concomitantly, further studies were conducted concerning the diffusion properties of these alloy types. The most recent studies among them, which applied the radiotracer method, found that there is no tendency of decreasing diffusivity with an increasing number of components, if compared on an absolute temperature scale [11].

An important aspect for a comprehensive understanding of bulk diffusion and radiation tolerance is the formation and migration behavior of vacancies in the multicomponent matrix. For this purpose, positron annihilation lifetime spectroscopy is a highly suitable technique, which is capable of detecting vacancy concentrations in the concentration range from $10^{-6}$ to $10^{-4}$ [12]. Positrons from a radioactive source are implanted into the sample, where they annihilate emitting two $\gamma$-quants. The positron lifetime in the material measured as the time difference between a start $\gamma$-quant $\left(\beta\right.$-decay of ${ }^{22} \mathrm{Na}$ ) and the annihilation $\gamma$-quant includes specific and sensitive information on the defect structure of the sample.

In a previous positron annihilation study [13] a high defect concentration was found in an as-cast $\mathrm{CoCrFeNi}$ alloy, but it could not be discerned whether these defects were dislocations or vacancies. On the other hand, for the case of as-cast $\mathrm{CoCrFeMnNi}$, it has been reported that the bulk of the material does not contain any structural defects [14]. In a recent study on the CoCrFeMnNi alloy, a strong increase of the mean positron lifetime with increasing temperature was observed indicating thermal generation of vacancies [15]. However, the positron lifetime in dependence of the temperature did 
not saturate, not even in the region of the highest applied temperature of $1473 \mathrm{~K}\left(0.92 T_{m, \mathrm{CoCrFeMnNi}}\right)$. Furthermore, the mean positron lifetimes measured at $293 \mathrm{~K}$ after the heat treatment were not significantly lower than the high temperature values which should be the case for reversible formation and removal of thermal vacancies. Hence, it remains unclear if the reported increase of the mean positron lifetime with increasing temperature is due to the thermal generation of vacancies or whether other processes in the multicomponent matrix such as phase decompositions play a role.

In the present study, positron annihilation lifetime spectroscopy is applied for the detection of thermally induced and quenched-in vacancies in a set of multicomponent concentrated solid-solution alloys. All of these alloys have a face-centered-cubic crystal structure, but exhibit an increasing number of constituents, from low to high mixing entropy. Well-defined samples of CrFeNi, CoCrFeNi, CoCrFeMnNi, and $\mathrm{AlCoCrFeNi}$ were subjected to an identical heat treatment under protective vacuum atmosphere, at high temperatures $(1493 \mathrm{~K})$, close to their onset of melting $T_{m}\left(T_{m, \mathrm{CoCrFeNi}}=\right.$ $1717 \mathrm{~K}, T_{m, \mathrm{CoCrFeMnNi}}=1607 \mathrm{~K}$ [11]). Subsequently the samples were rapidly cooled to $273.15 \mathrm{~K}$ by quenching them in ice water. Interestingly, the positron lifetime of the quenched samples shows a decreasing trend towards a defect-free bulk positron lifetime with an increasing number of constituents, i.e., with an increase in mixing entropy.

\section{EXPERIMENT}

All samples were produced from raw materials with a nominal purity better or equal to $99.95 \mathrm{wt} \%$. Master ingots with a mass of 20-30 g were initially produced by arc-melting in a zirconium-gettered argon atmosphere at 500 mbar absolute pressure. Every ingot was remelted six times before it was further processed. The alloys $\mathrm{CoCrFeNi}$ and $\mathrm{AlCoCrFeNi}$ were copper mold gravity cast into circular rods with a diameter of $8 \mathrm{~mm}$. After casting, each rod was recrystallized in an evacuated silica tube at $1273 \mathrm{~K}$ for $24 \mathrm{~h}$ and subsequently water quenched. The other alloy samples were prepared from pieces of the master ingots which were cold-rolled to a final thickness of about $1.5 \mathrm{~mm}$. The obtained sheets were heat treated in the same way as the cast rods. This process was chosen since the sheet shape facilitated the final sample preparation for the positron lifetime measurements.

Prior to any positron lifetime measurements, pairs of equivalent rectangular-shaped samples $(13 \mathrm{~mm} \times 5 \mathrm{~mm} \times 1.5 \mathrm{~mm})$ were sealed in evacuated silica tubes and kept at $1493 \mathrm{~K}$ for $30 \mathrm{~min}$. They were then rapidly quenched to $273.15 \mathrm{~K}$ by fast immersion and cracking of the silica tubes in ice water. Since all samples were still glowing bright yellow at the moment they came into direct contact with the ice water, their temperature can be estimated to be still at least $1373 \mathrm{~K}$ just before quenching. Using an approach as described in Ref. [16], the quenching rate for this procedure can be estimated to be at least $1200 \mathrm{~K} \mathrm{~s}^{-1}$. The samples were cooled to liquid-nitrogen temperatures immediately after this quenching procedure. For the positron lifetime measurements, the sample pairs were removed from the liquid-nitrogen bath and a ${ }^{22} \mathrm{Na}$ source encapsulated in aluminum foil was sandwiched between them. The source-sample arrangement was then transferred to the helium cryostat of the lifetime spectrometer, where it was kept at $205 \mathrm{~K}$ during the spectra acquisition. The time between the removal of the samples from the liquid-nitrogen bath until reaching subzero degrees in the helium cryostat was approximately $1 \mathrm{~min}$. The positron lifetime spectra were acquired using a digital lifetime spectrometer with a time resolution function of $174 \mathrm{ps}$ (full width at half maximum). Each lifetime spectrum contained more than $10^{6}$ counts and was analyzed after background and source correction $\left(\tau_{\text {source }}=332 \mathrm{ps}\right.$, $I_{\text {source }}=15 \%$ [17]) using the program PALSFIT [18].

In the recrystallized state, the crystal structure of all samples was investigated by X-ray diffraction (XRD) measurements. The XRD diffraction patterns were measured in $\theta / 2 \theta$ geometry using a Bruker D8 Advance equipped with a $\mathrm{Cu}$ anode $(\lambda=0.154 \mathrm{~nm})$. For data evaluation the software DIFFRAC.EVA was used. All samples were further characterized in the quenched state by electron backscatter diffraction (EBSD) and energy-dispersive X-ray spectroscopy (EDX). The EBSD and EDX measurements were conducted with a JEOL 7200F field-emission scanning electron microscope equipped with an Oxford Instruments EBSD/EDX system.

\section{RESULTS AND DISCUSSION}

The exact alloy compositions as obtained from EDX composition maps as well as the lattice constants as obtained from XRD measurements are given in Table I. Note that the compositions are further only given in the declarations of the nonequimolar alloys. Figure 1 shows the results of the EBSD and EDX mapping in the quenched state exemplary for the CoCrFeMnNi alloy. The EBSD phase mapping as shown in Fig. 1(a) clearly demonstrates the presence of only the facecentered cubic (fcc) phase in the quenched state of the alloy. It has to be noted, that the circumference of pores in the grains of the CoCrFeMnNi alloy are falsely detected as grain boundaries. The constituent distribution maps in Figs. 1(c)-1(g) show the homogeneous distribution of each element, with no evidence of any elemental segregation. Equivalent measure-

TABLE I. Alloy compositions in at. \% as obtained from EDX composition maps. The error of the alloy composition is determined to \pm 0.20 at. \%, and the error of the lattice constant is $0.001 \AA$.

\begin{tabular}{lccccccc}
\hline \hline Alloy & Al & Co & Cr & Fe & Mn & Ni & Lattice constant $a / \AA$ \\
\hline CrFeNi $(0.3: 1: 0.2)$ & & & 19.2 & 68.2 & & 12.6 & 3.581 \\
CrFeNi $(1: 1: 1)$ & & & 34.1 & 33.0 & & 3.9 & 3.560 \\
CoCrFeNi $(1: 1: 1: 1)$ & & 24.9 & 25.5 & 24.9 & & 24.7 & 3.570 \\
AlCoCrFeNi $(0.3: 1: 1: 1: 1)$ & 6.0 & 23.6 & 23.9 & 23.4 & & 23.1 & 3.588 \\
CoCrFeMnNi (1:1:1:1:1) & & 20.2 & 20.5 & 20.1 & 19.5 & 19.7 & 3.596 \\
\hline \hline
\end{tabular}




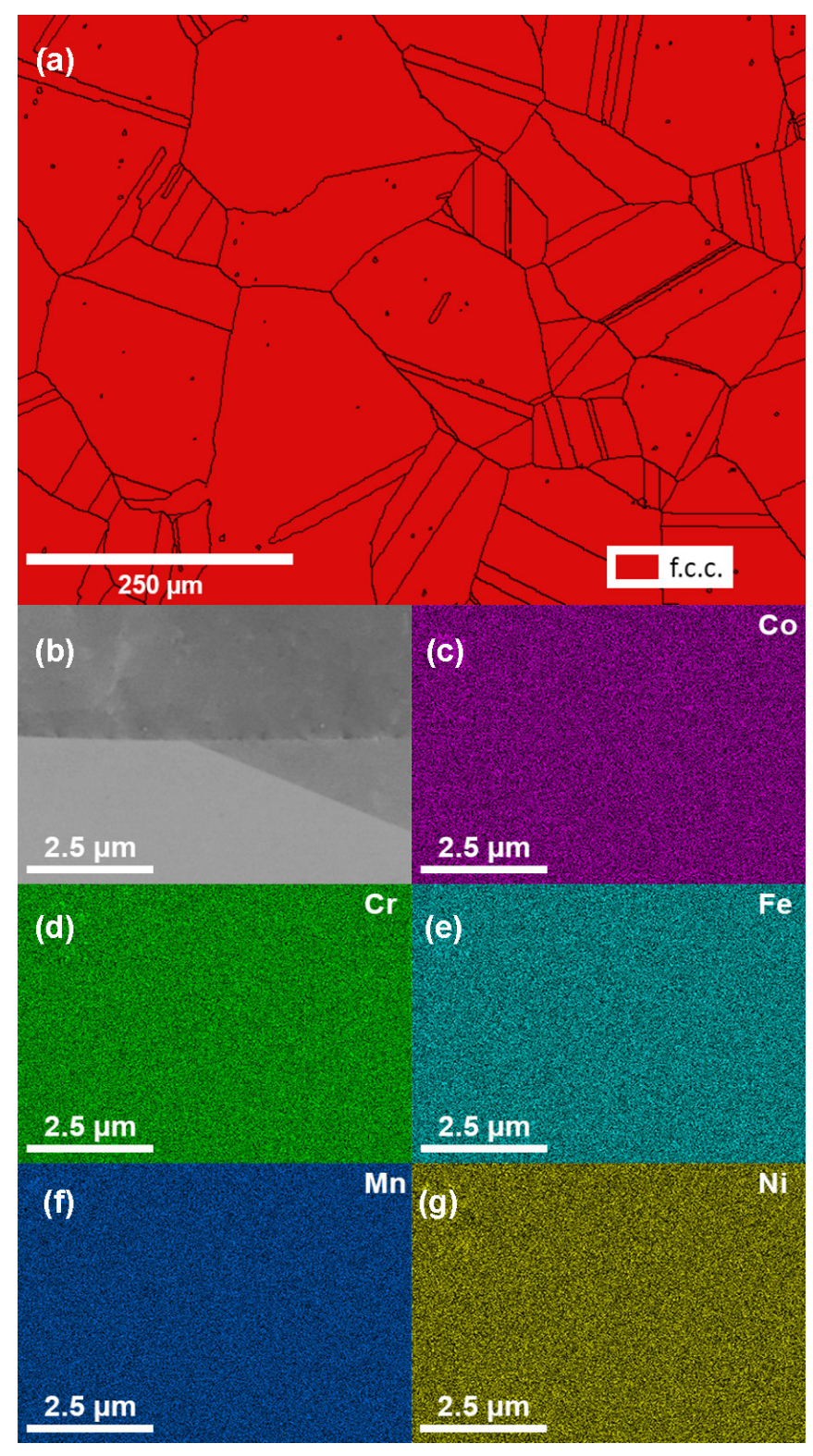

FIG. 1. EBSD and EDX measurements of the CoCrFeMnNi alloy in the quenched state. (a) EBSD phase mapping for a fcc phase with a lattice constant $a=3.596 \AA$. The circumference of pores inside the grains are falsely detected as grain boundaries. (c)-(g) Constituent distribution maps of the area shown in (b) for the alloy $\mathrm{CoCrFeMnNi}$ obtained by EDX mapping.

ments were conducted for all alloys investigated; in each case a single fcc phase and a homogeneous distribution of the constituents was observed (for all further EBSD and EDX results see the Supplemental Material [19]).

An analysis of the positron lifetime spectra was performed according to a two-state positron trapping model [20]. The two-state trapping model is generally valid for any type of material including one type of defect, which means that the experimentally observed positron lifetime spectrum consists of two exponential decays (after source correction). Hence, it is assumed that positrons annihilate either in a defect-free state exhibiting a lifetime $\tau_{\text {bulk }}$ or in the trapped state at a defect with a lifetime $\tau_{\text {defect }}=\tau_{2}$. The corresponding lifetime

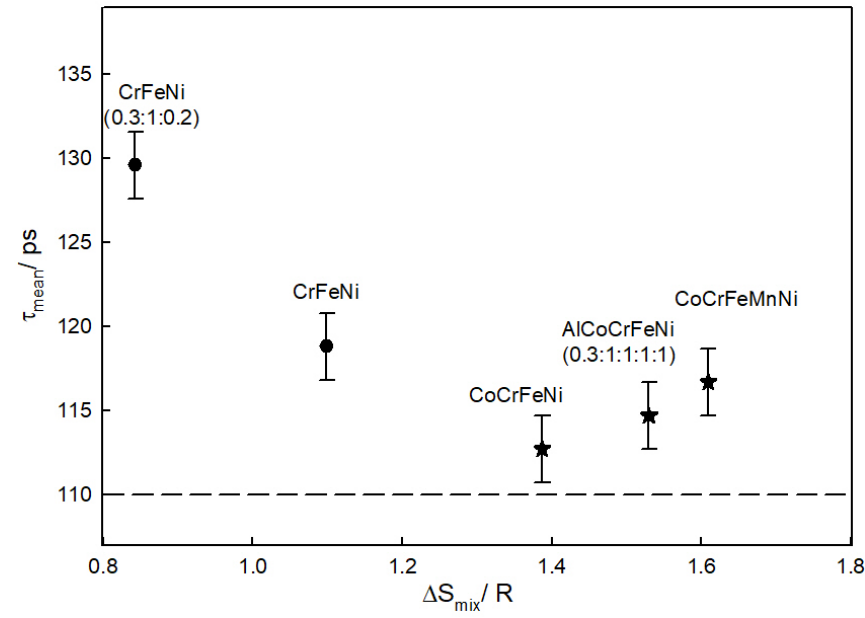

FIG. 2. Mean positron lifetime $\tau_{\text {mean }}$ as a function of the entropy of mixing $\Delta \mathrm{S}_{\mathrm{mix}}$. The mean lifetime $\tau_{\text {mean }}$ is obtained from twocomponent analysis (black dots) or corresponds to the value from the single-component analysis (black stars). The dashed line corresponds to the defect-free bulk lifetime of pure $\mathrm{Ni}$.

spectrum includes two components $\tau_{1}$ and $\tau_{2}$. From these the mean positron lifetime $\tau_{\text {mean }}$ is calculated according to $\tau_{\text {mean }}=$ $\tau_{1} I_{1}+\tau_{2} I_{2}$, where $I_{1}$ and $I_{2}$ are the corresponding intensities $\left(I_{1}+I_{2}=100 \%\right)$. The bulk lifetime $\tau_{\text {bulk }}$ attributed to the annihilation of positrons in the defect-free bulk, is calculated by $\tau_{\text {bulk }}=\left(\frac{I_{1}}{\tau_{1}}+\frac{I_{2}}{\tau_{2}}\right)^{-1}$. If there are no defects present only one lifetime component $\tau_{1}=\tau_{2}=\tau_{\text {bulk }}$ is detected.

In Fig. 2 the mean positron lifetime $\tau_{\text {mean }}$ for the set of alloys is shown as a function of their corresponding entropy of mixing $\Delta S_{\text {mix }}$ calculated according to $\Delta S_{\text {mix }}=-R \sum_{i} x_{i} \ln x_{i}$, with $x$ the mole fraction of constituent $i$ and $R$ the gas constant. For comparison, the defect-free bulk lifetime of pure $\mathrm{Ni}$ is also given in Fig. 1 (dashed line, $\tau_{\mathrm{Ni}}=110 \mathrm{ps}$ [21]). For the alloys CrFeNi (0.3:1:0.2) and $\mathrm{CrFeNi}$, the mean positron lifetime $\tau_{\text {mean }}$ is significantly higher than the expected, defect-free bulk lifetime. The mean positron lifetime decreases strongly with increasing mixing entropy $\Delta S_{\text {mix }}$ until it reaches a value slightly higher than the bulk lifetime of $\mathrm{Ni}$ as is the case for the alloys CoCrFeNi, AlCoCrFeNi (0.3:1:1:1:1), and CoCrFeMnNi. The enhanced mean positron lifetime observed in CrFeNi (0.3:1:0.2) and $\mathrm{CrFeNi}$ indicates the existence of thermally induced, quenched-in vacancies in these alloys as is typical for metals after quenching (see, e.g., Ref. [22]). Contrarily, the low mean positron lifetime of the alloys with higher entropy of mixing CoCrFeNi, AlCoCrFeNi (0.3:1:1:1:1), and $\mathrm{CoCrFeMnNi}$ does not indicate the presence of a significant amount of quenched-in vacancies, which is rather unexpected.

For a closer understanding of these results, a componentwise analysis of the positron lifetime spectra is given in Table II. It includes the entropy of mixing $\Delta S_{\text {mix }}$, the individual lifetime components $\tau_{1}\left(\tau_{2}\right)$, and the corresponding intensities $I_{1}\left(I_{2}\right)$ as well as the mean positron lifetime $\tau_{\text {mean }}$ and the bulk lifetime $\tau_{\text {bulk }}$ corresponding to the defect-free state. The confidence value of the best fits of the lifetime spectra as represented by the value of $\chi^{2}$ in Table II is excellent for all samples; $\chi^{2}$ even decreases towards higher mixing entropies. The positron lifetime spectra of the three-component alloys both contain two individual lifetime components $\tau_{1}$ and $\tau_{2}$ 
TABLE II. Mixing entropy $\Delta \mathrm{S}_{\mathrm{mix}}$, individual positron lifetime components $\tau_{1}\left(\tau_{2}\right)$, corresponding intensities $I_{1}\left(I_{2}\right)$, and calculated mean positron lifetime $\tau_{\text {mean }}$ and bulk positron lifetime $\tau_{\text {bulk }}$ of the individual sample pairs measured after the heat treatment. The errors for the individual lifetime components are given by the numerical uncertainty $\Delta \tau_{1 / 2}= \pm 2$ ps and $\Delta I_{1 / 2}= \pm 3 \%$, and the errors of the mean positron lifetime and the bulk positron lifetime are $\Delta \tau_{\text {mean } / \text { bulk }}= \pm 2 \mathrm{ps}$.

\begin{tabular}{lccccccc}
\hline \hline Alloy & $\Delta S_{\text {mix }} / R$ & $\tau_{1} / \mathrm{ps}$ & $I_{1} / \%$ & $\tau_{2} / \mathrm{ps}$ & $I_{2} / \%$ & $\tau_{\text {mean }} / \mathrm{ps}$ & $\tau_{\text {bulk }} / \mathrm{ps}$ \\
\hline CrFeNi $(0.3: 1: 0.2)$ & 0.84 & 104 & 72 & 195 & 28 & 130 & 120 \\
CrFeNi (1:1:1) & 1.10 & 117 & 97 & 190 & 3 & 119 & 118 \\
CoCrFeNi (1:1:1:1) & 1.39 & 113 & 100 & & & 113 & 1.169 \\
AlCoCrFeNi (0.3:1:1:1:1) & 1.53 & 115 & 100 & & & 115 & 1.131 \\
CoCrFeMnNi (1:1:1:1:1) & 1.61 & 117 & 100 & & & 117 & 1.110 \\
\hline \hline
\end{tabular}

with a similar bulk lifetime $\tau_{\text {bulk }}$ of 120 and 118 ps for $\mathrm{CrFeNi}$ $(0.3: 1: 0.2)$ and $\mathrm{CrFeNi}$, respectively. For the case of $\mathrm{CrFeNi}$ $(0.3: 1: 0.2)$ the best fit resulted in a long-lifetime component $\tau_{2}=195 \mathrm{ps}$ with an intensity of $I_{2}=28 \%$. For the fit of the lifetime spectrum of $\mathrm{CrFeNi}, \tau_{2}$ was held constant at a value of $190 \mathrm{ps}$, which is reasonable since both three-component alloys share the same fcc crystal structure and a very similar lattice constant (see Table I). For CrFeNi an intensity of $I_{2}=3 \%$ was found for the second lifetime component $\tau_{2}$. The theoretically calculated positron lifetimes in vacancies of the respective pure metals range from 184 to 201 ps [23]. Consequently, the second lifetime component $\tau_{2}$ of the three-component alloys has to be attributed to the annihilation of positrons in vacancies, which confirms the existence of quenched-in vacancies within these alloys.

Applying a two-state trapping model, the concentration of vacancies $c_{v}$ can be estimated from

$$
\mu_{d} c_{v}=\frac{I_{2}}{I_{1}}\left(\frac{1}{\tau_{\text {bulk }}}-\frac{1}{\tau_{2}}\right),
$$

where $\mu_{d}$ is the positron trapping rate coefficient [20]. For the $\mathrm{CrFeNi}(0.3: 1: 0.2)$ alloy, a concentration of quenched-in vacancies of $1.3 \times 10^{-5}$ can be estimated using a trapping rate coefficient $\mu_{d}=1 \times 10^{14} \mathrm{~s}^{-1}$. The equilibrium vacancy concentration $c_{v, e q}$ at a temperature $T$ can be calculated according to

$$
c_{v, e q}=\exp \left(\frac{S_{f}}{k_{B}}\right) \exp \left(-\frac{H_{f}}{k_{B} T}\right)
$$

with $k_{B}$ the Boltzmann constant, and $S_{f}$ and $H_{f}$ as the vacancy formation entropy and enthalpy. This results in an equilibrium vacancy concentration of $10^{-4}$ at $1493 \mathrm{~K}$ using values for $H_{f}$ and $S_{f}$ as reported for $\gamma$-Fe [24,25]. These results are in line, taking into account losses of a certain amount of vacancies during quenching.

On the other hand, the positron lifetime spectra of the alloys with four and five components $\mathrm{CoCrFeNi}, \mathrm{AlCoCr}-$ FeNi $(0.3: 1: 1: 1: 1)$, and CoCrFeMnNi exhibit only one single lifetime component. The obtained single lifetimes for $\mathrm{CoCr}-$ FeNi, AlCoCrFeNi (0.3:1:1:1:1), and CoCrFeMnNi were 113, 115, and $117 \mathrm{ps,}$, respectively. This slight increase of the positron lifetime $\tau_{1}$ is consistent with an increasing lattice constant as derived from XRD measurements (see Table I). In previous studies similar values for the positron lifetime in the defect-free bulk of CoCrFeNi ( $\tau_{\text {bulk }}=108$ ps [13] $)$ and $\mathrm{CoCrFeMnNi}\left(\tau_{\text {bulk }}=112\right.$ ps [14]) were found. Furthermore, the bulk lifetimes of the four- and five-component alloys are only slightly enhanced compared to the value for pure $\mathrm{Ni}$.
These deviations can also be explained due to differences in the packing densities of pure $\mathrm{Ni}$ and the investigated multicomponent alloys. Therefore, as already indicated by the mean positron lifetimes, also the componentwise analysis shows that no thermally generated vacancies can be detected in the alloys CoCrFeNi, AlCoCrFeNi (0.3:1:1:1:1), and $\mathrm{CoCr}-$ FeMnNi by the means of positron annihilation spectroscopy. It seems that vacancy concentrations in these samples are exceptionally low.

The question arises whether lattice vacancies cannot be detected due to strong competitive positron trapping at defects with short positron lifetimes. Shallow traps with positron lifetimes substantially shorter than that of lattice vacancies are considered for coherent and semicoherent precipitates in $\mathrm{Al}$ alloys [26]. Such types of traps can safely be excluded for these concentrated solid-solution alloys due to their singlephase homogeneous structure. Above all, the value of the single short positron lifetime $\tau_{1}$ observed in the four- and five-component samples (see Table II) corresponds to that expected for the free state.

Although any other interpretation of $\tau_{1}$ than the free state appears unlikely, for the sake of completeness, we may hypothetically discuss scenarios where $\tau_{1}$ would be associated with any kind of shallow trap. Assume, for instance, that $\tau_{1}$ represents a mean value of two components, one of which arises from a shallow type of trap with a characteristic positron lifetime $\tau_{\text {shallow }}=140 \mathrm{ps}$ and a relative intensity $I_{\text {shallow }}=40 \%$. For a mean value $\tau_{1}=115$ ps (AlCoCrFeNi sample, Table II), a value of 98 ps would follow for the other $60 \%$ component of the spectrum that is due to annihilation from the free state. These components ( 98 ps, 140 ps) both with substantial intensity should be clearly resolvable by numerical spectra analysis. This may no longer necessarily be the case if the intensity of $\tau_{\text {shallow }}$ is much lower and correspondingly the shorter component closer to $\tau_{1}$; however, this would also mean that the trapping rate of such shallow traps are much lower and, therefore, this would hardly hinder a detection of a positron lifetime component associated with vacancies, if present.

An extreme limit in this scenario would pertain to the case that the $\tau_{1}$ component arises from saturation trapping at shallow traps with $\tau_{\text {shallow }}=\tau_{1}$. Saturation trapping at such shallow traps indeed could mask competitive positron trapping at lattice vacancies. However, this can safely be ruled out, since there is neither a physical justification for positron traps with a lifetime as low as that of the free delocalized state, nor is there any reason for high concentration of such traps. An unambiguous proof that the absence of a vacancy-typical 
positron lifetime component does not arise from the existence of other traps, may be accomplished by measuring the positron diffusion length by means of low- and monoenergetic positron beams.

It can be concluded that the only consistent explanation for the nonobservation of the long-lifetime component in the quenched $\mathrm{CoCrFeNi}$, AlCoCrFeNi (0.3:1:1:1:1), and $\mathrm{CoCr}-$ FeMnNi samples is that they exhibit vacancy concentrations lower than $10^{-6}$ at that point. Even under the assumption that the slightly enhanced $\tau_{\text {mean }}$ compared to the value $\tau_{\text {bulk }}$ for $\mathrm{Ni}$ might originate from vacancies that cannot be resolved as separate components, an upper limit for the vacancy concentration of $7 \times 10^{-6}$ can be estimated. Therefore, there is either only a vanishingly small amount of vacancies being generated at temperatures close to their onset of melting, or the generated vacancies are inherently unstable and anneal out extremely fast. Both of these indications are completely unexpected for any densely packed multicomponent metallic crystal. Hence, also from this point of view, this type of ductile metallic material experiencing a very low or vanishingly small vacancy concentration can be regarded as a new class of material.

\section{CONCLUSION}

In the present study, the method of positron annihilation lifetime spectroscopy is applied to show that in the concentrated solid-solution alloys $\mathrm{CoCrFeNi}$, AlCoCrFeNi (0.3:1:1:1:1), and CoCrFeMnNi exhibiting a high entropy of mixing only a concentration of thermally generated vacancies smaller than $10^{-6}$ can be quenched-in. An identical heat treatment and quenching from temperatures close to their onset of melting was applied to a set of similar alloys with the same fcc crystal structure but with lower mixing entropy. For the latter case a vacancy concentration of $10^{-5}$ could be detected for $\mathrm{CrFeNi}$ (0.3:1:0.2). The mean positron lifetime of the samples decreases with increasing mixing entropy; furthermore, the lifetime spectra of the four- and five-component samples exhibit only one component. It can be concluded that in $\mathrm{CoCr}$ $\mathrm{FeNi}$, CoCrFeMnNi, and $\mathrm{AlCoCrFeNi}(0.3: 1: 1: 1: 1)$ there is either only a vanishingly small fraction of vacancies present at temperatures near the onset of melting or the generated vacancies are inherently unstable. Since in the latter case an unreasonably small migration enthalpy for the self-diffusion process must be assumed, the former explanation seems to be more promising.

\section{ACKNOWLEDGMENTS}

This work was performed in the framework of the interuniversity cooperation of TU Graz and Uni Graz on natural sciences (NAWI Graz). Support by the GIMRT Program of the Institute for Materials Research, Tohoku University (Grant No. 19K0513) is gratefully acknowledged.
[1] B. Cantor, I. Chang, P. Knight, and A. Vincent, Mater. Sci. Eng. A 375, 213 (2004).

[2] J.-W. Yeh, S.-K. Chen, S.-J. Lin, J.-Y. Gan, T.-S. Chin, T.-T. Shun, C.-H. Tsau, and S.-Y. Chang, Adv. Eng. Mater. 6, 299 (2004).

[3] High-Entropy Alloys, edited by M. C. Gao, J.-W. Yeh, P. K. Liaw, and Y. Zhang, (Springer International Publishing, Cham, 2016).

[4] E. P. George, D. Raabe, and R. O. Ritchie, Nat. Rev. Mater. 4, 515 (2019).

[5] Z. Li, K. G. Pradeep, Y. Deng, D. Raabe, and C. C. Tasan, Nature (London) 534, 227 (2016).

[6] C.-Y. Hsu, C.-C. Juan, W.-R. Wang, T.-S. Sheu, J.-W. Yeh, and S.-K. Chen, Mater. Sci. Eng. A 528, 3581 (2011).

[7] M.-H. Chuang, M.-H. Tsai, W.-R. Wang, S.-J. Lin, and J.-W. Yeh, Acta Mater. 59, 6308 (2011).

[8] J. J. Zhang, X. L. Yin, Y. Dong, Y. P. Lu, L. Jiang, T. M. Wang, and T. J. Li, Mater. Res. Innovations 18, S4-756 (2014).

[9] C. Lu, L. Niu, N. Chen, K. Jin, T. Yang, P. Xiu, Y. Zhang, F. Gao, H. Bei, S. Shi et al., Nat. Commun. 7, 1 (2016).

[10] F. Granberg, K. Nordlund, M. W. Ullah, K. Jin, C. Lu, H. Bei, L. M. Wang, F. Djurabekova, W. J. Weber, and Y. Zhang, Phys. Rev. Lett. 116, 135504 (2016).

[11] M. Vaidya, K. G. Pradeep, B. S. Murty, G. Wilde, and S. V. Divinski, Acta Mater. 146, 211 (2018).

[12] R. Krause-Rehberg and H. Leipner, Positron Annihilation in Semiconductors (Springer, New York, 1999).
[13] S. Abhaya, R. Rajaraman, S. Kalavathi, and G. Amarendra, J. Alloys Compd. 620, 277 (2015).

[14] M. Elsayed, R. Krause-Rehberg, C. Eisenschmidt, N. Eißmann, and B. Kieback, Phys. Status Solidi A 215, 1800036 (2018).

[15] K. Sugita, N. Matsuoka, M. Mizuno, and H. Araki, Scr. Mater. 176, 32 (2020).

[16] G. Adetunji, R. Faulkner, and E. Little, J. Mater. Sci. 26, 1847 (1991).

[17] Note that the source correction has been changed to $\tau_{\text {source }}=$ $293 \mathrm{ps}, I_{\text {source }}=13 \%$ for the $\mathrm{CrFeNi}(0.3: 1: 0.2)$ alloy.

[18] J. V. Olsen, P. Kirkegaard, N. J. Pedersen, and M. Eldrup, Phys. Status Solidi C 4, 4004 (2007).

[19] See Supplemental Material at http://link.aps.org/supplemental/ 10.1103/PhysRevMaterials.4.060601 for EBSD, EDX, and XRD results of all investigated alloys.

[20] P. Hautojärvi, A. Dupasquier, and M. Manninen, Positrons in Solids (Springer, New York, 1979), Vol. 12.

[21] G. Dlubek, O. Brummer, N. Meyendorf, P. Hautojarvi, A. Vehanen, and J. Yli-Kauppila, J. Phys. F 9, 1961 (1979).

[22] R. Cotterill, K. Petersen, G. Trumpy, and J. Traff, J. Phys. F 2, 459 (1972).

[23] M. Puska and R. Nieminen, J. Phys. F 13, 333 (1983).

[24] H. Matter, J. Winter, and W. Triftshäuser, Appl. Phys. 20, 135 (1979).

[25] J. Burton, Phys. Rev. B 5, 2948 (1972).

[26] B. Klobes, K. Maier, and T. Staab, Philos. Mag. 95, 1414 (2015). 